


\title{
12 la cooperación internacional en educación básica: el caso FEBLI
}

\author{
M arybel Zelaya, Olvis Castro
}

\section{INTRODUCCIÓN}

La educación constituye uno de los pilares fundamentales del desarrollo por lo que las sociedades actualmente han considerado invertir en este sector orientado ha aumentar la cobertura y a mejorar su calidad, en el caso particular de H onduras, el sector educación enfrenta serios problemas que inciden en las condiciones de subdesarrollo del país.

Es por ello que en el marco del curso de Políticas Internacionales de Cooperación para el Desarrollo se ha descrito un proyecto aplicado en educación a partir de 1994 hasta 2002 que tiene como nombre "Fomento a la Educación Básica en Lempira e Intibucá" (FEBLI), el cual se ejecutó con la ayuda técnica y financiera de la Cooperación A lemana, y ha tenido grandes beneficios para las comunidades en que fue aplicado.

EI proyecto FEBLI fue solicitado por la Secretaría de Educación de Honduras, a la Cooperación A lemana, con el propósito de mejorar la calidad de la educación básica y elaborar un currículo adecuado a las condiciones particulares de los departamentos de Lempira e Intibucá.

Se decidió hacer una descripción de los principales logros del FEBLI, por considerarse como uno de los proyectos más exitosos de la cooperación externa a la educación hondureña, especialmente en el campo de la adecuación curricular.

En la elaboración de este informe una de las tareas principales fue caracterizar la situación educativa hondureña, revisar los planteamientos de las conferenciasinternacional es sobre educación, identificar los tipos y modal idades de cooperación, esto nos permitió obtener un marco de referencia para seleccionar el proyecto que aquí se describe .

\section{CARACTERIZACIÓN DE LA SITUACIÓN EDUCATIVA EN HONDURAS}

En los últimos años se han dado al gunos avances en educación sobre todo en el nivel primario. El analfabetismo ha disminuido en más de 20 puntos durante los últimos 30 años; el promedio de escolaridad ha aumentado de 2.3 A ños en 1974 a 4.6 años y la tasa neta de matrícula de educación primaria aumentó de $78 \%$ en 1980 a $86.7 \%$ en 1999. En 1997 el crecimiento de esta matrícula es mayor en el área rural ( $2.5 \%$ anual) que en el área urbana(1.5\%) (ERP, 2001).

A pesar de los avances que ha tenido la educación en nuestro país no podemos pasar por alto las grandes dificultades que aun persisten, es por ello que la Secretaria de Educación presentó en el año 2000 una propuesta que permita la tran sformación del sistema educativo del país, la cual se fundamenta en los siguientes problemas:

\section{a. Cobertura insuficiente}

Los siguientes datos nos reflejan la situación educativa en cuanto a cobertura que brinda el Estado a nivel de educación pre escolar, primaria y secundaria:

b. Inadecuada formación de los docentes

El actual docente de educación primaria recibe su formación inicial en las escuelas normales que son, hasta ahora instituciones de nivel medio en contraste con la gran mayoría de los países Latinoamericanos y de otros países donde los docentes se forman en el nivel universitario 0 en todo caso post secundaria.

c. Baja C alidad del Proceso Enseñanza A prendizaje

La deficiente calidad educativa es el problema esencial del sistema. La baja calidad se refleja en la relevancia y pertinencia de los aprendizajes, en las altas tasas de deserción y repetición sobre todo en lostres primeros grados y en el bajo rendimiento y logro académico de los alumnos y las al umnas. En educación primaria la repetición es de $9.75 \%$ y la deserción es de $3.4 \%$; en educación media la repetición es de $10.31 \%$.

La baja calidad está relacionada además, con la inadecuada formación inicial y permanente de los y las docentes en servicio, carencia de infraestructura adecuada, de materiales educativos, de equipos pedagógicos y recursos tecnológicos, existe un currículo cen-

\section{Cuadro No. 1}

\begin{tabular}{|lcc|}
\hline Nivel Educativo & $\begin{array}{c}\text { Cobertura } \\
\text { Neta en } \%\end{array}$ & $\begin{array}{l}\text { Cobertura } \\
\text { Bruta en } \%\end{array}$ \\
\hline Pre-Escolar & 38.85 & sd. \\
Primaria & 86 & 99.25 \\
Secundaria & 21.89 & 35 \\
\hline
\end{tabular}

Fuente: Secretaría de Educación, Transformación del Sistema Educativo en Honduras .\{Propuesta dela Secretaría de Educación

Cobertura Neta se refiere a la población en edades comprendidas para cada nivel escolar I. Cobertura Bruta: se refiere a la población que no está comprendida en los rangos de edad para cada nivel, pero que está matrículada. 
tralizado que no responde a las necesidades del educando, la familia la comunidad y el país, y que no forma al educando para el futuro.

d. Desarticulación del Sistema Educativo

$\mathrm{N}$ o existe una estructura que articule los diferentes niveles, ciclos y modalidades educativas. $\mathrm{N}$ o se ha definido adecuadamente el nivel de educación básica, especialmente con la incorporación del tercer ciclo $\left(7^{\circ}, 8^{\circ}\right.$ y $9^{\circ}$ grados) y su articulación con los restantes niveles. Por otra parte no se ha logrado un enfoque que desarrolle el concepto de educación para el trabajo en los diferentes niveles, ciclos y modalidades que garantice que el egresado del sistema educativo tenga los conocimientos y destrezas básicas para insertarse con eficiencia en el sistema laboral.

e. Insuficiente Tiempo del A lumno en el A ula.

La ausencia de los docentes es significativa en todos los niveles educativos. En educación primaria, de los 200 días que deberían laborar, apenas cubren 110 días al año. Este problema es mayor en áreas rurales. A demás, el $62 \%$ de los maestros en educación primaria enseñan en escuelas multigrado, esto supone un menor tiempo de atención y dedicación a los alumnos por grado.

f. Escasa A tención a la Diversidad Etnica.

En los grupos étnicos ( $G$ arífunas, Lencas, C hortís, M isquitos, Tahuakas, Pech, Tolupanes, Isleños etc) la lengua materna no es el español; por lo tanto, es necesario establecer programas bilingües en los centros educativos ubicados en esas comunidades.

Dadas la situación de la multiculturalidad, los niños y niñas que pertenecen a las diferentes etnias, están en desventaja con los demás al tener que hacer uso de materiales educativos escritos en español y no en su lengua materna.

g. Inadecuada Supervisión y Evaluación Educativa.

Este es otro elemento que incide de manera determinante en la baja calidad porque no se realiza un seguimiento y evaluación del desempeño del docente, ni se conoce a profundidad el rendimiento académico de los y las alumnos (as). Existe un débil control de asistencia de los maestros. Con la puesta en marcha del proceso de desconcentración de la educación, el problema de la supervisión se ha profundizado especialmente en educación media, porque las tareas que realizaban los supervisores fueron delegadas a personal técnico de cada Dirección D epartamental y D istrital , sin la preparación necesaria para desempeñar estas funciones.

h. Deficiente A decuación del Currículo.

U no de los problemas básicos en la educación es el referido al currículo, el cual es elaborado por personal técnico a nivel central. En el caso de la educación primaria fue elaborado un plan de estudios en la primeros años de la década de los 50 y sufrió modificaciones en 1967 con la comisión de reforma de planes y programas, y en 1988 al establecerse los ren dimientos básicos.

El nivel medio tiene planes de estudios que varían según las modalidades. El plan del Ciclo Común de C ultura G eneral fue elaborado en 1959 y ha sufrido pequeñas reformas, pero no han sido suficientemente modificados. i. Limitaciones Financieras e Instalaciones Físicas I nadecuadas.

Este es un problema grave que se detecta en todos los centros, especial mente en los rural es que suponen el $67 \%$ de las escuelas donde las condiciones prevalecientes son la carencia de agua potable, electricidad y servicio sanitario.

j. Escasa Eficiencia Terminal.

Las altas tasas de deserción y repetición inciden en el hecho de que un bajo porcentaje de alumnos en el nivel primario finalice sus seis años de estudio. En una cohorte a nivel nacional en 1997, se estimó que de cada 1000 alumnos matriculados solamente 528 finalizan el sexto grado, lo que significa una eficiencia terminal muy baja.

k. Insuficiente Educación de A dultos.

En 1996, se atendió en las escuelas primarias aceleradas un total de 84,088 alumnos atendidos en 3,999 instituciones. El 88\% de la matrícula correspondió al sector formal y $12 \%$ a programas de Ias ONGS. En esta actividad se destaca el programa de EDU CATODOS que atendió el 39.7 \% y el Programa de A Ifabetización y Educación Básica de Jóvenes y A dultos (PRA LEBA H), que desde 1997 ha atendido la cantidad de 44,000 alumnos sobre una expectativa de 65,000 al finalizar el proyecto. En este campo se necesita prestar mayor atención porque el analfabetismo en nuestro país es muy elevado; actualmente se estima en un 19\%.

\section{Escasa Educación Especial}

A pesar de que aproximadamente $10 \%$ de la población hondureña presenta algún tipo de discapacidad, la cobertura en servicios de educación especial es apenas del 0.1\%. La cantidad de instituciones públicas es menor que la del sector privado. Gran parte de las instituciones están ubicadas en Tegucigalpa y San Pedro Sula

m. Falta de Desarrollo del M odelo de G estión A dministrativa

La estructura administrativa de la Secretaría de Educación, a pesar de haberse creado lasD irecciones D epartamentalesy D istritales, todavía no ha alcanzado el nivel de eficiencia requerido en la prestación de los servicios educativos, por cuanto no están claramente definidas las funciones para cada nivel (central, departamental, distrital o local). A ún subsisten, a nivel central ciertas tareas técnicas que deberían trasladarse a la Direcciones D epartamentales . En este sentido, es fundamental el mejoramiento de los procedimientos y controlesadministrativos a fin de lograr mayor eficiencia del sistema.

n. Inadecuado M arco Legal.

El sistema educativo tiene instrumentos legales obsoletos e inadecuados que entran en contradicción con la constitución de la República, la Ley de Educación Superior, El Estatuto del Docente y otras leyes aprobadas recientemente.

\section{Impacto del H uracán Mitch y Aspectos Claves del Sector}

Los mayores daños sufridos en este sector se manifiestan en 2,465 aulas escolares afectadas a nivel nacional, de las cuales 787 
fueron destruidas 1,678 resultaron con daños parciales. Los datos incluyen a 123 cocinas escolares; 105 bodegas, 701 módul os sanitarios y 1473 letrinas. En general, se estima que los daños a las instalaciones, equipo y mobiliario de los centros afectados (incluyendo la educación superior) ascienden a U S\$ 19.6 millones.

A demás, fue severamente afectado el edificio de la Secretaría de Educación, cuya reconstrucción se estimó en $U S \& 6.3$ millones Finalmente, también se agregan U $\$ \$ 6.2$ millones que se derivan de daños a los centros escolares y otras instituciones como el IN FO P ,al servir de al berguesa los damnificados, y por reubicación temporal de las escuelas.

Se estima que unos 100,000 alumnos fueron afectados, Io cual provocó deserción e inasi stencia a clases. Esto puede estar asociado a la reducción de los ingresos a nivel familiar y a la falta de una alimentación adecuada; además es muy probable que los daños en la infraestructura vial dificulten la movilidad de una buena parte de los alumnos, sobre todo en las regiones más aisladas. Debido a ello, en el corto plazo los esfuerzos por aumentar el acceso al sistema educativo podrían verse reducidos a las tasas de cobertura similares a la década anterior.

Los cambios e impactos mencionados se presentan en un contexto educativo que atraviesa por una crisis profunda que afecta a los diferentes sectores del sistema educativo nacional. Entre los principales problemas cabe destacar:

- La alta incidencia de la pobreza. Esto limita la asistencia a las escuelas y el rendimiento académico de niños y niñas de familias de menores recursos

- El acceso a la educación. Solamente uno de cada cinco en edad correspondiente ingresa a la educación pre escolar; y menos de la mitad de los que egresan del sexto grado accesan al nivel básico de educación secundaria. Esto hace difícil lograr la meta establecida en el estudio sectorial -Plan Decenal de 1999 , relativo a que el $50 \%$ de la fuerza laboral pueda terminar su educación secundaria aunado a ello se encuentran problemas de rendimiento académico y repetición de los alumnos que afectan severamente el sistema educativo nacional .

- Falta de eficiencia en la educación superior. A ctualmente se presentan algunos problemas en los esquemas administrativos y legales que dirigen la educación superior los cual es están vinculados a la presencia de diversos enfoques e intereses que requieren ser armonizados en función de las necesidades que demanda el país. Esta situación se traduce en deficiencias de aspecto académico; descuido de la investigación científico tecnológica teórica y aplicada, y una débil relación de retroalimentación con el medio circundante.

\section{CONFERENCIAS MUNDIALES SOBRE EDUCACIÓN}

\section{a. Conferencia Educación para Todos realizada en Jomtiem Tailandia 1990}

En 1990, en Jomtiem (Tailandia), se adquiere el compromiso, por parte de todos los gobiernos del mundo, de conseguir la alfabetización universal plena para el año 2.000, ya que se ha calculado que existen 125 millones de niños sin posibilidad de ir a la escuela y otros 150 que abandonarán la escuela antes de terminar los cuatro años necesarios para adqui rir unos conocimientos básicos y perdurables.

En esta conferencia se plantearon los siguientes objetivos

1. A mpliación del cuidado y el desarrollo del niño en la primera infancia.

2. A cceso universal a la educación primaria y terminación también universal antes del año 2000.

3. Reducción a la mitad de losnivel es correspondientesa 1.990 en cuanto al analfabetismo adulto, con especial atención a la alfabetización de las mujeres.

4. M ejora de los resultados del aprendizaje, basada en el logro de unos determinados niveles previamente establecidos.

5. Expansión de la educación básica y la capacitación para jóvenes y adultos.

6. M ejora en la difusión del conocimiento, las habilidades y los valores necesarios para un desarrollo sostenido.

\section{b. Conferencia Sobre Población y D esarrollo realizada en El Cairo 1994}

Según la C onferencia de Población y Desarrollada realizada en El Cairo en 1994, respecto a la educación de la población, se plantearon los siguientes objetivos:

a. Lograr el acceso de todos (as) a una enseñanza de calidad dando especial prioridad a la enseñanza primaria y técnica y la capacidad para el empleo, la lucha contra el analfabetismo y la eliminación de la desigualdad entre los sexos con respecto al acceso, la retención y el apoyo a la educación.

b. Incorporar en los programas de estudio temas sobre la relación entre la población y desarrollo sostenible, las cuestiones de salud, incluida la salud reproductiva y la igual dad entre los sexos, mejorando su contenido a fin de fomentar una mayor responsabilidad y conciencia al respecto.

\section{c. Conferencia de El Cairo + 5 realizada en 1999}

En la conferencia de El C airo + 5 realizada en 1999 se propuso Io siguiente:

1. Debería procurarse especialmente aumentar las tasa de permanencia de las niñas en la escuela primaria y secundaria, para tal fin habría que sensibilizar a los padres respecto de la importancia de la educación de los hijos en particular de las niñas para que estas alcancen su pleno desarrollo.

2. Los gobiernos, en particular de países en desarrollo con la asistencia de la comunidad internacional deben:

2.1 A mpliar las políticas y los programas de educación de jóvenes y adul tos y de aprendizaje permanente que tengan en cuenta las cuestiones de género prestando mayor atención a los migrantes, los 
pueblos indígenas y las personas discapacitadas.

2.2 Reducir la tasa de an alfabetismo de niñas y niños de modo que llegue a más de la mitad para las/los niñas y niños en el 2005, en comparación con la tasa de 1990.

2.3 Proporcionar instalaciones debidamente dotadas mediante la rehabilitación de las escuelas existentes y la construcción de otras nuevas.

\section{d. Conferencia sobre Educación realizada en Dakar, año 2000}

En esta conferencia se plantearon las siguientes metas:

a. Expandir y mejorar el cuidado infantil y la educación inicial integral, especialmente para los niños y niñas más vul nerables y en desventaja.

b. A segurar que para el 2015 todos los niños y niñas, especialmente en circunstancias difíciles accedan y completen una educación primaria gratuita, obligatoria y de buena calidad.

c. A segurar la satisfacción de las necesidades de aprendizaje de jóvenes y adultos a través del acceso equitativo a programas apropiados de aprendizaje de habilidades para la vida y la ciudadanía.

d. M ejorar en un $50 \%$ los niveles de alfabetización de adultos para el 2015, especialmente dentro de las mujeres y lograr acceso equitativo a la educación básica y permanente para todas las personas adultas.

e. Eliminar las disparidades de género en educación primaria y secundaria para el 2005, y lograr la equidad de género para el 2015 asegurando a las niñas el acceso a una educación básica de calidad y rendimientos plenos e igualitarios.

f. M ejorar todos los aspectos de la calidad de la educación y asegurar la excelencia de todos (as), de modo que logre resultados de aprendizaje reconocidos y medibles, especial mente en torno a la alfabetización, el cálculo y las habilidades esenciales para la vida

\section{ESTRATEGIA DE REDUCCIÓN DE LA POBREZA PARA HONDURAS}

Es una política de Estado con visión a largo plazo y dentro de sus metas globales en cuanto a educación plantea lo siguiente:

1. Lograr una cobertura neta del $70 \%$ en el tercer ciclo de la educación básica $\left(7^{\circ}\right.$ a $\left.9^{\circ}\right)$ Para ello será necesario, además de realizar un incremento real del presupuesto de la Secretaría de Educación, mejorar la calidad educativa tanto en la formación de docentes como en el currículo; ampliar los incentivos como becas o subsidios directos que ayuden a retener a los estudiantes de hogares pobres en losúltimos tres años de educación básica, con participación comunitaria.

2. Lograr que el $50 \%$ de la nueva fuerza laboral complete la educación secundaria, en este caso también será importante mejorar la calidad educativa, ampliar y desarrollar incentivos que permitan a estudiantes continuar los siguientes años de la educación se- cundaria, especialmente en colegios técnicos, aumentando de manera sustancial la oferta de centros escolares y lograr mayor participación de las 0 N Gs, empresarios, padres de familia etc.

\section{LA COOPERACIÓN INTERNACIONAL EN EL CASO DE HONDURAS.}

La cooperación para el desarrollo es una inversión fundamental para la mejora del bienestar económico y social en los países en desarrollo y promueve la aparición de nuevos socios económicos cada vez mas dinámicos (CA D, 2000)

La cooperación internacional ha orientado gran parte de sus ayudas en el sector educación, por considerarse fundamental para el desarrollo de los países.

Bajo esta perspectiva $\mathrm{H}$ onduras ha considerado la educación como una prioridad nacional donde el recurso humano se constituye en la base del desarrollo productivo y tecnológico del país y por ende esto influye en la mejora de la calidad de vida de la población.

N uestro país ha sido beneficiado por la cooperación internacional en los diferentes sectores, en el caso particular de la educación, dicha cooperación se ha presentado a nivel bilateral y multilateral y ha impactado en forma positiva en el desarrollo del país sobre todo a nivel social.

Dentro de la cooperación bilateral recibida por $\mathrm{H}$ ondurasuna de las más destacadas ha sido la proporcionada por A lemania.

Honduras fue elegida por el Gobierno alemán como uno de los países prioritarios de la cooperación intergubernamental de la República Federal de A lemania. Las metas generales de la misma son: la reducción de la pobreza y el fomento del desarrollo sostenible. Para lograr estas metas, la Cooperación Técnica y Financiera trabaja en tres sectores prioritarios:

1. Política ambiental, conservación y uso sostenible de los recursos naturales.

2. Educación.

3. Reforma económica y desarrollo de la economía de mercado.

Para efectos de este trabajo, nuestro mayor interés está dirigido al sector educación en el cual la cooperación alemana, a través de la GTZ desarrolla el programa de Educación y Desarrollo Social (PRODES) cuyo objetivo es apoyar la transformación de la educación hondureña, con calidad y relevancia.

Entre sus proyectos se encuentran los siguientes:

* A sesoría a la Secretaria de Educación (A SED)

* Fomento a la Educación Básica (FEBLI)

* Formación Inicial de Docentes (PRO FID)

* Educación para todos (Cooperación con el Banco M undial)

* A sesoría al G abinete Social. 


\section{ESTUDIO DE CASO}

Este estudio ha tenido a bien describir el proyecto "Fomento a la Educación Básica en los Departamentos de Lempira e Intibucá " (FEB LI)

El cuadro N o. 2 muestra la matriz general del proyecto.

M ejorar la calidad de la educación primaria contribuyendo a incrementar la participación comunitaria, adecuar el currículo a la realidad educativa de la región, mejorar el funcionamiento de la administración escolar y contribuir al mejoramiento de la estructura

\section{a. Antecedentes del proyecto}

Los problemas de educación primaria en los departamentos de Lempira e Intibucá son, en gran medida, los mismos que tiene la educación primaria en todo el país: baja calidad, insuficiente cobertura, altas tasas de deserción y repitencia, baja eficiencia terminal.

Estos problemas, sin embargo, han presentado una mayor gravedad en estos dos departamentos, explicable por el atraso de su economía, su topografía montañosa, las condiciones de pobreza de la mayoría de su población, las particularidades de su mestizaje y el marginamiento de la población indígena, mantenida en una situación secular de supervivencia.

Dadas sus características geográficas sociales y culturales, tienen especial significación los problemas relacionados con el escaso tiempo dedicado a la enseñanza aprendizaje, la renuencia de los maestros a trabajar en el sector rural, el bajo rendimiento en las asignaturas básicas, especialmente en español y matemáticas, el predominio de las metodologías frontales, la práctica inveterada de los maestros de no desarrollar aquellos temas considerados difíciles y el escaso desarrollo de los contenidos propios de las $\mathrm{C}$ iencias $\mathrm{N}$ aturales y de las Ciencias Sociales.

Basado en estos antecedentes, la Secretaria de Educación solicitó a la C ooperación A lemana su A sistencia Técnica para la Educación de dicho proyecto, a partir de junio de 1994 con el propósito de mejorar la calidad de la educación básica y elaborar un currículo adecuado a las condiciones particulares de dichos departamentos.

EI FEBLI se ha ejecutado en tres fases, cada una de ellas con su objetivo y logros, en el cuadro No. 3 se detallan con mayor precisión.

\section{b. Principales logros del proyecto FEB LI}

1. Se ha adecuado el currículo de Ciencias $\mathrm{N}$ atural es a las condiciones locales de estos departamentos

Como resultado del proceso de la adecuación curricular se ha diseñado y validado una experiencia innovadora que parte del saber pedagógico de los maestros y de las condiciones sociocul tural es de la zona. El eje fundamental de este proceso de adecuación curricular a las condiciones socioculturales de la zona es la asignatura de Español.

2. Consolidación de una estrategia de capacitación y mejoramiento de la labor docente.

Esta experiencia de adecuación curricular ha sido en riquecida a través de las capacitaciones desde el espacio donde tiene lugar el que hacer pedagógico del maestro o capacitaciones sobre ejes temáticos de pedagogía y gestión. La estrategia en si se consolidó a lo largo del proceso.

Cuadro No. 2

\begin{tabular}{|c|c|c|c|c|}
\hline Fuente & $\begin{array}{l}\text { Nombre } \\
\text { del Proyecto }\end{array}$ & Descripción & $\begin{array}{l}\text { Inicio/ } \\
\text { termino }\end{array}$ & $\begin{array}{c}\text { Monto } \\
\text { exterior en dólares }\end{array}$ \\
\hline Alemania & $\begin{array}{l}\text { Fomento a la } \\
\text { Educación Básica } \\
\text { en Lempira e } \\
\text { Intibuca }\end{array}$ & $\begin{array}{l}\text { Mejorar la calidad de la } \\
\text { educación primaria contribu- } \\
\text { yendo a incrementar la } \\
\text { participación comunitaria, } \\
\text { adecuar el currículo a la } \\
\text { realidad educativa de la } \\
\text { región, mejorar el funciona- } \\
\text { miento de la administración } \\
\text { escolar y contribuir al mejora- } \\
\text { miento de la estructura }\end{array}$ & $\begin{array}{l}\text { Junio de } \\
1994 \text { a } \\
\text { diciembre } \\
\text { del } 2002\end{array}$ & $490,196.00$ \\
\hline
\end{tabular}

Fuente: SETCO,Matriz del Proyecto FEBLI. 2002 


\section{Cuadro No. 3. Fomento a la Educación Básica en Lempira e Intibucá}

\begin{tabular}{|c|c|c|c|}
\hline FECHA & FASES & OBJETIVOS & LOGROS \\
\hline $\begin{array}{l}\text { Junio de } 1994 \\
\text { a diciembre de } \\
1997\end{array}$ & $\begin{array}{l}\text { I. Planifica- } \\
\text { ción estraté- } \\
\text { gica del } \\
\text { proyecto } \\
\text { FASES }\end{array}$ & $\begin{array}{l}\text { * Mejorar la calidad } \\
\text { de } \\
\text { La educación básica } \\
\text { en los departamen- } \\
\text { tos de Lempira e } \\
\text { Intibucá }\end{array}$ & $\begin{array}{l}\text { 1. Diagnóstico de la situación educativa socioeconómica y cultural de } \\
\text { la zona. } \\
\text { 2. Diseño y validación de conceptos pedagógicos innovadores. } \\
\text { 3. Lineamientos validados para la participación de la comunidad en el } \\
\text { proceso educativo. } \\
\text { 4. Manual de talleres para el fomento de la lectura. } \\
\text { 5. Adecuación del currículo de Ciencias Naturales y Español a la } \\
\text { realidad de los departamentos de Intibucá y Lempira. } \\
\text { 6. Selección de Escuelas pilotos para desarrollar la experiencia según } \\
\text { las necesidades obtenidas en el diagnóstico departamental. } \\
\text { 7. Dotación de equipo,mobiliario y reacondicionamiento de las } \\
\text { escuelas pilotos. } \\
\text { 8. Dotación de mobiliario a las oficinas. }\end{array}$ \\
\hline $\begin{array}{l}\text { Enero de } 1999 \\
\text { a diciembre del } \\
2000\end{array}$ & $\begin{array}{l}\text { II Consolida- } \\
\text { ción de } \\
\text { Resultados }\end{array}$ & $\begin{array}{l}\text { Aplicar los enfoques } \\
\text { y métodos validados } \\
\text { en el ámbito nacional }\end{array}$ & $\begin{array}{l}\text { 1. Estrategias de sostenibilidad y difusión de las experiencias exitosas } \\
\text { del proyecto. } \\
\text { a. Establecimiento de alianzas estratégicas con otros programas } \\
\text { proyectos e instituciones con componente educativo mediante } \\
\text { convenios, acuerdos y coordinaciones. } \\
\text { b. Funcionamiento de equipo técnico de gestión departamental, } \\
\text { distrital y local. } \\
\text { 2. Desarrollo de Métodos. } \\
\text { a. Elaboración y aplicación de la serie Manuales de } \\
\text { "Capacitación Docente". } \\
\text { 3. Producción de libros y manuales educativos, para docentes y } \\
\text { alumnos en la experiencia de adecuación curricular. } \\
\text { 4. Participación en el proceso de reconstrucción de escuelas, en el } \\
\text { Programa de Emergencia, después del huracán Mitch (se constru- } \\
\text { yeron } 22 \text { aulas escolares). }\end{array}$ \\
\hline $\begin{array}{l}\text { Enero del } \\
2001 \text { a } \\
\text { diciembre del } \\
2002\end{array}$ & $\begin{array}{l}\text { III. Difusión y } \\
\text { sostenibilidad }\end{array}$ & $\begin{array}{l}\text { Difundir a nivel } \\
\text { nacional enfoques, } \\
\text { métodos e } \\
\text { instrumentos } \\
\text { validados en la fase } \\
\text { anterior }\end{array}$ & $\begin{array}{l}\text { 1. Aprovechamiento de los métodos y enfoques de la experiencia del } \\
\text { proyecto FEBLI, desarrollados en Lempira e Intibucá en el proceso } \\
\text { de trasformación educativa de la Secretaría de Educación. } \\
\text { 3. Difusión y divulgación de las experiencias exitosas del proyecto en } \\
\text { otros departamentos del país. } \\
\text { a. Capacitación de docentes en la metodología } \\
\text { aplicada en la experiencia. } \\
\text { b. Distribución de materiales educativos elaborados en el } \\
\text { proyecto. } \\
\text { c. Coordinación y establecimiento de alianzas estratégicas, con } \\
\text { otras organizaciones e instituciones del departamento y a nivel } \\
\text { nacional. } \\
\text { 4. Asesorar a las Direcciones Departamentales de Educación (DDE) de } \\
\text { Intibucá y Lempira, para una gestión eficiente en su departamento. } \\
\text { a. Capacitación a Directores Departamentales y Distritales en } \\
\text { planificación operativa y estratégica. } \\
\text { b. Asesoramiento para el funcionamiento de organismos de } \\
\text { estructura que den sostenibilidad a las acciones administrati- } \\
\text { vas y técnicas. } \\
\text { c. Asesoramiento a las administraciones distritales y departamen- } \\
\text { tales para la elaboración y ejecución de sus planes de segui- } \\
\text { miento y evaluación de la gestión. }\end{array}$ \\
\hline
\end{tabular}


Esta estrategia ha llevado a los maestros a :

- Reflexionar sobre su rol no sólo de orientadores del proceso enseñanza aprendizaje y del contexto en el cual se desarrolla sino también de investigadores, Ejemplo: la identificación de dificultades en los cuadernos oficiales de trabajo delos niñosy niñas en el aprendizaje de la escritura; transformadores del mismo al proponer y validar la alternativa de solución a dichas dificultades, es decir, adecuar el currículo real a dicho contexto.

- La elaboración y validación de materiales educativos adecuados al contexto real del trabajo del maestro.

- Redescubrir potencialidades en sus alumnos y alumnas antes no consideradas: Iosniños aprenden y recrean un nuevo conocimiento y también aprenden como hacer conocimiento. Con ello losniños(as) se convierten en participantes del proceso de adecuación curricular.

- Integrar y enriquecer los contenidos de capacitación desarroIlados a través de otras estrategias, al proceso de enseñanza aprendizaje mismo.

\section{La gestión educativa ha mejorado}

Este proceso se inició dentro del contexto de reestructuración y desconcentración de la Secretaría de Educación. Para su sostenibilidad se partió de brindar asesoría a nivel central hasta llegar a nivel local, es decir a la gestión escolar. Este proceso se resume en:

- A poyo a la Secretaría de Educación en el proceso de reestructuración y desconcentración de la misma.

- Experiencia piloto de capacitación sobre elaboración del Plan O perativo A nual (POA), con la participación e integración de los distintos niveles educativos, en coordinación con el IN ICE.

- Elaboración Plan O perativo A nual y seguimiento - Evaluación Escolar-POA con participación de las distintas fuerzas vivas de la comunidad estructuras organ izativas comunitarias, instituciones y $O N$ G s que desarrollan proyectos en la comunidad.

- Diseño, desarrollo y seguimiento de proyectos de ampliación y reparación del edificio escolar por parte de la comunidad en coordinación con ON G s, construcciones escolares y la municipalidad. Estos proyectos, además de mejorar las condiciones educativas de la escuela capacitan la comunidad en la gestión de proyectos (Ejemplo identificación y aprovechamiento de recursos organizacionales, institucional es y humanos), lo cual a la vez fortalece otras áreas de la vida comunal, contribuye al mejoramiento de las condiciones de vida de la misma

c. Escuelasy Población beneficiada a través del proyecto FEBLI

Cuadro No. 4. Escuelas de Influencia Directa en el Proyecto FEBLI, año 1996

\begin{tabular}{lllll|}
\hline \multicolumn{2}{c}{ Departamento de Lempira } \\
\hline 12 Escuelas & 12 aldeas & 8 municipios & 851 alumnos & 20 docentes \\
Marco Aurelio Soto & Cerro Colorado & San Manuel & 65 & 1 \\
Juan Rafael Mora & El Cacahuatal & La Virtud & 51 & 2 \\
República de Brasil & El Ciprés & San Manuel & 90 & 1 \\
Tecauxinas & Santa Catarina & La Campa & 112 & 1 \\
Armando Escalón & Cubite & San Sebastián & 62 & 1 \\
15 de Septiembre & El Coral & & 97 & 2 \\
Juan Castillo & Barrio Nuevo & Erandique & 110 & 2 \\
Honduras & San Isidro & Santa Cruz & 58 & 1 \\
Guillermo Muñoz & Santa Rosita & & 69 & 2 \\
Cabañas & Santa Rita & Mapulaca & 35 & 1 \\
Ramón Villeda Morales & San José & La Virtud & 52 & 2 \\
Martín López & El Rodeo & Virginia & 50 & 2 \\
La Democracia & & & & 2 \\
\hline
\end{tabular}


Cuadro No. 5. Departamento de Intibucá

\begin{tabular}{|c|c|c|c|c|}
\hline \multicolumn{5}{|c|}{ Departamento de Intibucá } \\
\hline 12 Escuelas & 12 ald eas & 8 municipios & 898 alumnos & 20 docentes \\
\hline España & El Apintal & Colomoncagua & 117 & 2 \\
\hline Romualdo Bueso Peñalba & Los Amates & & 55 & 1 \\
\hline Independencia & San Antonio del Norte & Camasca & 58 & 2 \\
\hline Francisco Morazán & Los Pozos & Magdalena & 77 & 2 \\
\hline Exequel Romero & Calusica & Concepción & 94 & 2 \\
\hline Juan Lindo & Monte Verde & & & \\
\hline San Francisco de Opalaca & & & 163 & 3 \\
\hline Pedro Nufio & & Santa Cruz & 85 & 2 \\
\hline España & San Lorenzo & & 35 & 1 \\
\hline \multirow[t]{2}{*}{ Francisco Morazán } & Panina & & & \\
\hline & & Intibucá & 48 & 1 \\
\hline Presentación Centeno & El Naranjal & & 55 & 1 \\
\hline José Cecilio del Valle & San Bartolomé & & 41 & 1 \\
\hline Manuel de Jesús Subirana & San Juan & San Antonio & 70 & 2 \\
\hline
\end{tabular}

\section{CONCLUSIONES}

- Nuestro país ha enfrentado grandes dificultades, en el caso de la educación las carencias más notables han sido: cobertura insuficiente, inadecuada formación de docentes, baja calidad del proceso enseñanza apren dizaje, desarticulación del sistema educativo, insuficiente tiempo del alumno en el aula, escasa atención a la diversidad étnica, inadecuada supervisión y evaluación educativa, deficiente adecuación del currículo, limitaciones financieras e instalaciones físicas inadecuadas, escasa eficiencia terminal, insuficiente educación de adultos, escasa educación especial, falta de desarrollo del modelo de gestión educativa e inadecuado marco legal. Toda la problemática antes mencionada ha motivado a la cooperación internacional a invertir en este sector y a través del proyecto FEBLI en particular ha contribuido de alguna manera solventar en parte esta situación.

- FEBLI ha llevado a cabo un proceso de desarrollo curricular para las asignaturas de $\mathrm{Ci}$ iencias $\mathrm{N}$ aturales, A gropecuaria y Español con un enfoque pedagógico y metodológico que es aplicable a las demás áreas de aprendizaje. Este proyecto ha impactado de tal manera que las experiencias sirvieron para diseñar el nuevo C urrículo N acional Básico (CN B), con valideza nivel nacional, el que será implementado a partir del 2004.
- Con el apoyo del proyecto se ha establecido la práctica de planificar anual mente las actividades a nivel escolar, distrital y departamental, lo que ha estimulado la participación de los actores sociales y ha aumentado el compromiso para alcanzar los objetivos. De igual manera se ha capacitado técnicamente el personal docente de los diferentes niveles tomando como base la experiencia del proyecto.

- La metodología experimentada a través del proyecto ha sido reconocida como aporte de gran valor para el mejoramiento de la calidad de la educación básica, logrando que el modelo didáctico metodológico sea compartido con convicción por docentes, al umnos y padres de familia.

- Las actividades del proyecto han tenido un impacto significativo en la promoción del desarrollo a través de un trabajo muy concreto desde las bases y en coordinación con otras instituciones para contribuir al mejoramiento de la educación básica en los departamentos de alta pobreza, como ser Lempira e Intibucá. 